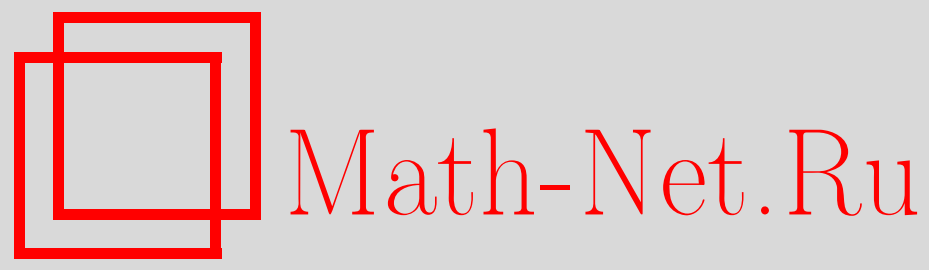

В. А. Треногин, Теорема Люстерника и наилучшая параметризация решений нелинейных уравнений, Функи. анализ и его прил., 1998, том 32, выпуск 1, 87-90

DOI: https://doi.org/10.4213/faa407

Использование Общероссийского математического портала MathNet.Ru подразумевает, что вы прочитали и согласны с пользовательским соглашением

http://www . mathnet.ru/rus/agreement

Параметры загрузки:

IP : 18.209 .158 .208

26 апреля 2023 г., 06:11:33

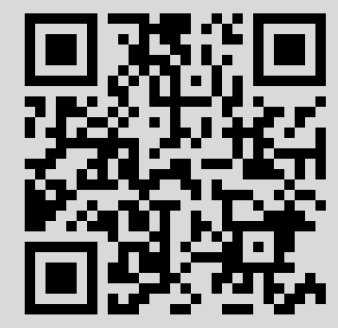




\title{
Теорема Люстерника и наилучшая параметризация решений нелинейных уравнений ${ }^{\star}$
}

\author{
(C) 1998. В. А. ТРЕногин
}

Пусть $X$ и $Y$ - вещественные банаховы пространства, и пусть оператор $F(x)$ отображает окрестность $S$ точки $x_{0}$ в окрестность нуля пространства $Y$, причем $F\left(x_{0}\right)=0$. Пусть оператор $F(x)$ удовлетворяет следующим дополнительным предположениям.

I. $F(x)$ дифференцируем на $S$ в смысле Фреше, и его производная $F^{\prime}(x)$ удовлетворяет на $S$ условию Липшица с постоянной $c>0:\left\|F^{\prime}\left(x_{1}\right)-F^{\prime}\left(x_{2}\right)\right\| \leqslant$ $c\left\|x_{1}-x_{2}\right\|$.

II. Область значений $R(B)$ оператора $B=-F^{\prime}\left(x_{0}\right)$ совпадает с $Y$.

III. Множество нулей (ядро) $N=N(B)$ оператора $B$ обладает в $X$ прямым дополнением $U$, т.е. существует проектор $P$, такой, что $N=P X$, $U=(I-P) X$.

Заметим, что в этих условиях (см. [2]) оператор $B_{U}$, являюшийся сужением на $U$ оператора $B$, отображает $U$ на $Y$ взаимно однозначно и $\Gamma=\left(B_{U}\right)^{-1} \in$ $L(Y, X)$.

Рассмотрим уравнение $F(x)=0$. Его решение будем искать в следующем виде:

$$
x=x_{0}+\varphi+u, \quad \varphi \in N, u \in U .
$$

Здесь мы считаем $\varphi$ малым параметром, а $u$ неизвестным элементом, т.е. исходное уравнение трактуется как уравнение, определяющее неявный оператор.

Введем следующие обозначения:

$$
m=\|\Gamma\| \cdot c, \quad \beta=(\sqrt{2}-1) / m, \quad \alpha=(1-1 / \sqrt{2}) / m, \quad q=1 / \sqrt{2} .
$$

Теорема 1. Пусть выполнены условия I-III. Тогда при каждом $\varphi c$ $\|\varphi\| \leqslant \beta$ в шаре $\|u\| \leqslant \alpha$ существует единственное решение $u=u(\varphi)$ уравнения $F\left(x_{0}+\varphi+u\right)=0$, причем $u(0)=0$. Решение $и(\varphi)$ дифферениируемо при $\|\varphi\|<\beta$, причем $u^{\prime}(0)=0$, и его можно найти прочессом последовательных приближений:

$$
u_{n+1}(\varphi)=u_{n}(\varphi)-\Gamma F\left(x_{0}+\varphi+u_{n}(\varphi)\right), \quad n=0,1,2 \ldots, u_{0}(\varphi)=0 .
$$

Справедлива оченка скорости сходимости

$$
\left\|u_{n}(\varphi)-u(\varphi)\right\| \leqslant q^{n} / m .
$$

Доказательство проводится по схеме, предложенной нами в [1, п. 36.4]. При этом $q$ подобрано так, чтобы $u$ было определено в шаре наибольшего радиуса. 
СлЕДСТвиЕ 1. Существует гладкое многообразие решений уравнения $F(x)=0$ вида (1) с $и=u(\varphi)$, определяемьм теоремой 1.

ЗАмечАниЕ. Результат следствия является вариантом известной теоремы Люстерника (см. [3, гл. VII, 10]). Ниже будет показано, что предложенная им параметризация многообразия решений с помощью параметров, лежащих в касательном многообразии, является наилучшей с нескольких точек зрения.

Попробуем теперь параметризовать решения уравнения $F(x)=0$ с помощью параметров из некоторого подпространства $M$ в $X$, удовлетворяющего такому ограничению:

IV. Пусть $M$ - замкнутое подпространство в $X$, имеющее в $X$ прямое дополнение $V$, т. е. существует проектор $Q$, такой, что $M=Q X, V=(I-Q) X$.

На проектор $Q$ мы налагаем следующее существенное условие:

V. Сужение $Q_{N}$ оператора $Q$ на подпространство $N$ отображает $N$ на $M$ взаимно однозначно.

Следующее важное предложение лежит в основе дальнейшего изложения.

Лемма 1. Пусть $B_{V}$ - сужение оператора $B$ на подпространство $V$. Если выполнены ограничения IV $u \mathrm{~V}$, то $B_{V}$ отображает $V$ на $Y$ взаимно однозначно, оператор $\Gamma_{V}=\left(B_{V}\right)^{-1}$ лежит в $L(Y, X)$ и решение уравнения $B v=y, v \in V$, дается формулой

$$
v=\Gamma_{V} y=\left(I-\left(Q_{N}\right)^{-1} Q\right) \Gamma y,
$$

где $Q_{N}$ - сужение оператора $Q$ на $N$.

Возвратимся к уравнению $F(x)=0$. Теперь его решения мы будем искать в виде

$$
x=x_{0}+e+v, \quad e \in M, v \in V .
$$

Введем следующие сокращенные обозначения:

$$
\mu=\left\|\Gamma_{V}\right\| \cdot c, \quad q(k)=\left(k^{2}+k+\frac{1}{2}\right)^{1 / 2}-k, \quad \rho=\frac{2 q(k)-1}{\mu}, \quad r=\frac{1-q(k)}{\mu},
$$

где $k=\left\|\Gamma_{V}\right\|\left\|B_{M}\right\|$, а $B_{M}-$ сужение $B$ на $M$.

Tеорема 2. Пусть выполнень условия I, II, IV $u$ V. Тогда при каждом $е$ из шара $\|e\| \leqslant \rho$ в шаре $\|v\| \leqslant r$ существует единственное решение $v=v(e)$ уравнения $F\left(x_{0}+e+v\right)=0$, оно непрерьвно дифференцируемо при $\|e\|<\rho u$ $v(0)=0$. Решение $v=v(e)$ можно получить прочессом последовательных приближений

$$
v_{n+1}(e)=v_{n}(e)-\Gamma_{V} F\left(x_{0}+e+v_{n}(e)\right), \quad n=0,1,2, \ldots, \quad v_{0}(e)=0 .
$$

Справедлива оченка скорости сходимости

$$
\left\|v_{n}(e)-v(e)\right\| \leqslant q^{n}(k) / \mu .
$$

СлЕДСТВИЕ 2. Уравнение $F(x)=0$ имеет гладкое семейство решений (4) c $v=v(e)$, определяемьм теоремой 2. Если $v^{\prime}(0)=0$, mо $M=N$.

ЗАмЕчАниЕ. $q(k)$ и $\rho(k)$ являются строго убывающими функциями параметра $k$, а $r(k)$ — строго возрастающей его функцией. Если $M=N$, то $k=0, \mu=m$ и теорема 2 превращается в теорему 1 . 
ЗАмЕЧАНИЕ. Если условие $\mathrm{V}$ нарушено, то возможно, что или $R\left(B_{V}\right) \neq Y$ или $N\left(B_{V}\right) \neq\{0\}$, или то и другое одновременно. Здесь неудачный выбор параметров продолжения обычно приводит к ветвлению решения (см. [2]).

В заключение остановимся на случае гильбертова пространства $X$. Условия III и IV мы заменим теперь такими условиями:

$\mathrm{III}_{1} . P$ - ортопроектор пространства $X$ на $M, U=(I-P) X$.

$\mathrm{IV}_{1}$. $M$ замкнуто в $X, Q$ - ортопроектор пространства $X$ на $M, V=$ $(I-Q) X$.

Приведем сначала несколько элементарных предложений, позволяющих легко проверить выполнение условия V. В леммах 2-4 $X$ - гильбертово пространство.

ЛЕмма 2. Условие $\mathrm{V}$ выполнено, если одновременно выполнены условия 1) для любого $\varphi \in N$ существует $e$, такое, ито $(\varphi, e) \neq 0, u 2)(I-P) M \subset$ $(I-P) V$. Если, в частности, $N$ и $M$ одномерны, $\varphi-$ базисный элемент в $N$, , е - базисньй элемент в $M$, то условие $\mathrm{V}$ равносильно тому, что $(\varphi, e) \neq 0$.

Лемма 3. Пусть $N$ и $M$ суть $n$-мернье пространства с базисами $\varphi_{i}$, $i=1, \ldots, n, u e_{j}, j=1, \ldots, n$, соответственно. Если $\operatorname{det}\left(\varphi_{i}, e_{j}\right) \neq 0$, то условие $\mathrm{V}$ выполнено.

Лемма 4. Если $\varphi_{i}, i=1, \ldots, n,-$ базис в $N, a Q \varphi_{i}, i=1, \ldots, n,-$ базис в $М$, то условие $\mathrm{V}$ выполнено.

Из формулы (3) и теоремы Пифагора в $X$ вытекает следующее предложение:

ЛЕмМА 5. Пусть выполнены условия I, II, $\mathrm{III}_{1}, \mathrm{IV}_{1}, \mathrm{~V}$. Тогда имеет место оченка $\|\Gamma\| \leqslant\left\|\Gamma_{V}\right\|$. Равенство достигается при $M=N$.

ТЕорема 3. В условиях леммы 5 выбор параметров продолжения в касательном многообразии $x_{0}+N \kappa$ многообразию решений исходного уравнения позволяет определить это многообразие решений в шаре наибольшего радиуса. Иначе говоря, наибольшее значение $\rho=\rho(M)($ см. (5)) по всевозможньлм подпространствам $M$, удовлетворяюшим условию $\mathrm{V}$, достиааетсл при $M=N$.

Доказательство следует из замечания к теореме 2 и леммы 5.

ТЕОРемА 4. Оченка абсолютной погрешности вычисления на каждом шаге последовательньх приближений в теореме 2 будет наилучшей, если параметры продолжения выбраны в касательном многообразии.

ДоказАтельство. Рассмотрим точное уравнение $B_{V} v=y$ и приближенное уравнение $C w=z$ с оператором $C \in L(V, Y)$. Пусть $\left\|B_{V}-C\right\| \leqslant \delta$, $\|y-z\| \leqslant \delta$. Рассмотрим всевозможные подпространства $V$, удовлетворяющие условию $\left\|\Gamma_{V}\right\| \delta<1$. Аналогично п. 22.3 из [1] имеем оценку $\|w-v\| \leqslant$ $\Delta\left(\left\|\Gamma_{V}\right\|\right)$, где $\Delta(d)=\left(d \delta+d^{2}\|y\| \delta\right)(1-\delta d)^{-1}$. Функция $\Delta(d)$ строго возрастает, а из леммы 5 следует, что ее минимум достигается при $V=U$, т.е. при $M=N$.

ЗАмЕчАНиЕ. В случае однопараметрического многообразия решений речь идет о длине дуги кривой решений как наилучшем параметре продолжения. 
Этот путь был предложен в [4], а затем численно реализован и обоснован в ряде конкретных ситуаций в духе теоремы 4 (см., например, [5, 6]).

Получим оценку меры обусловленности оператора $B_{V}$, позволяющую вывести оценку относительной погрешности вычисления точного решения (см. $[1$, п. 22.2]). Для этого введем в предположении V следующую численную характеристику близости подпространств $M$ и $N: p(M)=\left\|\left(Q_{N}\right)^{-1} Q(Q-P)\right\|$.

Заметим, что $p(N)=0$. Кроме того, $p(M)$ может принимать сколь угодно большие значения. Так будет, например, если $X$ гильбертово, а в $M$ и в $N$ имеются ненулевые элементы, как угодно близкие к ортогональным.

ТЕОРЕмА 5. В условиях леммы 5 имеет место следуюшая оченка мерь обусловленности $\mu\left(B_{V}\right)$ оператора $B_{V}: \mu\left(B_{V}\right) \leqslant \mu\left(B_{U}\right)\left(1+p^{2}(M)\right)^{1 / 2} u$, таким образом, оченка относительной погрешности на каждом шаге последовательных приближений будет наименьшей при $M=N$.

Доказательство проводится так же, как и в теореме 4 (см. [1, п. 22.4]); оно использует теорему Пифагора в $X$ и определение $p(M)$.

Здесь мы продолжили исследование различных аспектов метода продолжения по параметру (см. [7-8]). Случай однопараметрического семейства решений был анонсирован нами в [9].

\title{
ЛИТЕРАТУРА
}

1. Треногин В. А. Функциональный анализ. Наука, М., 1980, 1993. 2. Вайнбера M. M., Треногин B. A. Теория ветвления решений нелинейных уравнений. Наука, М., 1969. 3. Люстерник Л. А., Соболев В. И. Элементы функционального анализа. Наука, М., 1965. 4. Ворович И. И., Зипалова В. Ф. Прикл. матем. и мех., 29, вып. 5, 894-901 (1965). 5. Григолюк Э. И., Шалашилин В. М. Проблемы нелинейного деформирования. Наука, М., 1988. 6. Шалашилин В. И., Кузнецов Е. Б. Докл. РАН, 334, № 5, 566-568 (1994). 7. Треногин В. А. Функц. анализ и его прил., 30, вып. 2, 93-95 (1996). 8. Треногин В. А. Докл. РАН, 350, № 4, 455-457 (1996). 9. Треногин $B$. A. В кн.: Тезисы международной конференции "Актуальные проблемы механики и сплошных и сыпучих сред». Москва, 1997, с. 127.

Московский государственный институт стали и сплавов (технологический университет)

Поступило в редакцию 17 апреля 1997 г.

УДК 517.41

\section{Следы операторов рождения-уничтожения и формулы Фредгольма}

\author{
(c) 1998. A. B. ЧЕРвов
}

Доказана формула для следов некоторого класса операторов (к нему, в частности, относятся вертексные операторы), следы которых могут быть использованы для вычисления корреляционных функций и формфакторов в

* Работа выполнена при поддержке Российского фонда фундаментальных исследований, проект 96-15-96939, и INTAS, грант 93-0183. 